\title{
Efecto de edad relativa en la Liga Nacional de Básquetbol de Chile (LNB)
}

\author{
Relative Age Effect in Chile's National Basketball League (LNB)
}

\author{
*Hernán Andrés Salinas Molina
}

Salinas, H. (2021). Efecto de edad relativa en la Liga Nacional de Básquetbol de Chile (LNB). Revista Ciencias de la Actividad Física UCM, N²2(1), enero-junio, 1-11. DOI: http://doi.org/10.29035/rcaf.22.1.7

\section{RESUMEN}

El objetivo del estudio fue verificar la existencia de sobrerrepresentación de jugadores nacidos en la primera parte del año o Efecto de Edad Relativa (EER), en la elite del baloncesto masculino chileno.

A partir de las nóminas oficiales de la Liga Nacional de Básquetbol de Chile (LNB) para la temporada 2019-20, se obtuvieron las distribuciones por mes, trimestre y semestre, de las fechas de nacimiento de los jugadores. Estas fueron comparadas con las distribuciones de nacimientos en Chile entre 1977 y 2005. La significancia estadística de las diferencias entre distribuciones esperadas y observadas se verificó mediante la prueba de $\chi^{2}$ (mensual y trimestral) y una distribución binomial (semestral).

Los tres análisis arrojaron diferencias significativas entre las distribuciones esperadas y observadas, con una sobrerrepresentación de los nacidos en el primer semestre, primer trimestre y el mes de enero y subrepresentación de los nacidos el segundo semestre, cuarto trimestre y mes de diciembre. Se concluye que hay evidencia suficiente para confirmar la existencia de EER en LNB.

Para disminuir el EER se proponen medidas tendientes a educar a entrenadores acerca de los efectos de las diferencias de edad en el rendimiento y el establecimiento de fechas de corte móviles durante la temporada competitiva.

Palabras clave: Efecto de edad relativa, Baloncesto, Liga Nacional de Básquetbol, Edad cronológica, Edad biológica, Identificación de talentos.

\section{ABSTRACT}

The objective of the study was to verify the existence of overrepresentation of players born in the first part of the year or Relative Age Effect (RAE), in Chilean elite men's basketball.

The distribution by month, quarter, and semester of players' birthdates were obtained from the official rosters of the National Basketball League of Chile (LNB) for the 2019-20 season. These were compared to the distribution of births in Chile between 1977 and 2005. The statistical significance of the differences between expected and observed distribution was verified using the $\mathrm{\square} 2$ test (monthly and quarterly) and a binomial distribution (semi-annual).

The three analyses yielded significant differences between the expected and observed distribution, with an overrepresentation of those born in the first semester, first quarter, and January and an underrepresentation of those born in the second semester, fourth quarter, and December. It is concluded that there is sufficient evidence to confirm the existence of RAE in LNB.

To reduce RAE, measures are proposed to educate coaches about the effects of age differences on performance and the establishment of mobile cut-off dates during the competitive season.

Key words: Relative Age Effect, Basketball, National Basketball League, Chronological Age, Biological Age, Talent Identification.

* Institute for Globally Distributed Open Research and Education (IGDORE). Universidad Nacional Andrés Bello: Santiago, Chile. https://orcid.org/0000-0002-8846-8104 


\section{Planteamiento del problema}

Los deportes de situación son actividades donde las cualidades cognitivas, coordinativas, condicionales y antropométricas, son determinantes para el éxito deportivo (Lorenzo, 2002). En jóvenes, el desarrollo de estas cualidades está fuertemente influenciado por el nivel de maduración y edad del deportista.

Entre los jóvenes, los deportistas mayores presentan generalmente mejores rendimientos cognitivos, coordinativos y condicionales, por lo que es esperable que los niños mayores tengan niveles de rendimiento deportivo más altos que sus pares de menor edad (Delorme et al., 2010).

En el deporte formativo, atendiendo a las naturales ventajas de los mayores, en las competencias los deportistas son agrupados de acuerdo con un criterio de edad cronológica, es decir, se establece que todos los jugadores nacidos entre ciertas fechas límite, competirán en una categoría determinada. Estas fechas corresponden normalmente al primer y último día de un año calendario (Gutierrez et al., 2010, Barnsley \& Thompson, 1988).

Esta forma de organización permite que se generen diferencias de hasta un año en la edad de los participantes dentro de una misma categoría, lo que favorecería que los jugadores mayores, es decir, nacidos justo después de la fecha de corte, tengan una mayor probabilidad de ser percibidos como "talentos deportivos", debido a que su mayor edad les permitiría alcanzar mayores niveles de rendimiento (Addona \& Yates, 2010).

Las ventajas de una mayor edad determinan mayor facilidad para conseguir resultados precoces e integrarse a programas deportivos y de esta forma, el deportista puede ser considerado por su entorno como una "estrella precoz" (Manonelles et al., 2003).

Estas diferencias de edad al interior de una categoría pueden resultar en el retiro voluntario de los más jóvenesy/o en una promoción formal de los mayores a niveles superiores de entrenamiento y competencia. Estos dos fenómenos entregan a los jugadores nacidos justo después de la fecha de corte, mayores chances de iniciar carreras deportivas, lo que, entre deportistas de elite, se manifiesta como una sobrerrepresentación de los nacidos durante los primeros meses del año (Delorme et al., 2009), fenómeno conocido como Efecto de Edad Relativa (EER) (Isorna et al., 2014).

Esta sobrerrepresentación ha sido documentada en diversos deportes y contextos, entre los que se cuentan, el judo en los JJOO (Albuquerque et al., 2015), programas de tecnificación de piragüismo en España (Isorna et al., 2014), hockey sobre hielo en Canadá (Barnsley \& Thompson, 1988), la ATP (Loffing et al., 2010), el balonmano alemán (Schorer et al., 2013) y el fútbol profesional europeo (úbeda et al., 2020).

El baloncesto no es ajeno al EER. Un estudio realizado en los campeonatos mundiales de la FIBA de las categorías U17, U19 y U21 entre los años 1979 y 2011, arrojó diferencias significativas entre una distribución uniforme y la distribución de jugadores varones por trimestre de nacimiento, con una sobrerrepresentación de los nacidos el primer trimestre del año (34,32\% en vez del 25\% esperado) y una subrrepresentación de los nacidos durante el cuarto trimestre (14,83\%) (Saavedra et al., 2015).

También se ha documentado el fenómeno en el baloncesto juvenil europeo. Los jugadores participantes en el torneo U18 "Adidas Next Generation Tournament", en las temporadas 2013-2014 y 20142015 ( $n=581$ ), fueron incluidos en un estudio que encontró en ambas temporadas que alrededor del 68\% de los jugadores nacieron durante el primer semestre; (72,1\% y $71,7 \%$ de los bases en la primera y segunda temporada respectivamente, 66,6\% y 71,4\% de los aleros y 61,8\% y 60,9\% de los centros). Este estudio además encontró correlaciones estadísticamente significativas entre indicadores de rendimiento y edad de los jugadores. Bases y centros mayores anotan más puntos y toman más tiros de 2 puntos que los más jóvenes (Ibáñez et al., 2018).

Otro estudio realizado entre seleccionados españoles, encontró que en las categorías Cadete, Juvenil y Junior, los nacidos en T1, T2, T3 y T4 eran un 
$54 \%, \quad 28,8 \%, \quad 8,5 \%$ y $8,5 \%$ respectivamente (Manonelles et al., 2003).

A nivel de clubes, una investigación estudió el fenómeno a través de la distribución de las fechas de nacimiento de jugadores que abandonaron la práctica formal durante la temporada 2006-2007, según los registros de la Federación Francesa de Básquetbol (FFBB). Los resultados mostraron subrepresentación de abandonos entre los jugadores nacidos en T7 y T2 y sobrerrepresentación de abandonos entre aquellos nacidos en T4, en las categorías de "9 - 10 años", "11 - 12 años y "13 - 14 años" y el primer año de la categoría "15 - 17 años" (Delorme et al., 2011).

Por otro lado, existen estudios donde se contradice lo anterior y no se ha encontrado evidencia del fenómeno en baloncesto. Por ejemplo, en 2016 se encontró que el EER no estaba presente entre los jugadores de baloncesto participantes en los JJOO de Londres, salvo entre los jugadores franceses (Werneck et al., 2016).

\section{Objetivos de la Investigación}

En Chile, no ha sido posible encontrar artículos que aborden el fenómeno de EER. Sin embargo, considerando que las fechas de corte para las categorías formativas y la estructura de las competencias son similares en todo el mundo, el presente estudio pretende ser una primera aproximación al EER en Chile, persiguiendo como objetivo el verificar su existencia entre los jugadores participantes en la principal liga de baloncesto masculino del país, Liga Nacional de Básquetbol (LNB).

\section{Diseño metodológico}

Para establecer las distribuciones esperadas de las fechas de nacimiento, se recopilaron los Anuarios de Demografía de 1977 a 1996 (exceptuando 1979 que no estaba disponible) a través del Sistema Integral de Información y Atención ciudadana (SIAC) del Instituto Nacional de Estadísticas (INE). Además, desde el sitio web del INE (s.f.) se recopilaron Anuarios de Demografía de 1997 a 1999 y los Anuarios de Estadísticas Vitales de 2000 a 2005. De estos documentos se extrajo la cantidad de nacimientos registrados en el territorio chileno entre 1977 y 2005 distribuidos por mes de ocurrencia, con los que se construyó la distribución de frecuencia relativa esperada por mes, trimestre y semestre de nacimiento.

Desde el sitio web de estadísticas de LNB, (Febachile, 2019) se recopilaron las nóminas oficiales de los equipos participantes en la temporada 20192020 con sus respectivas fechas de nacimiento. De un total de 267 registros, se eliminaron los registros de jugadores extranjeros (70), sin nacionalidad (13) y de chilenos sin fecha de nacimiento (1) quedando finalmente un total de 183 registros ( $n=183$ ).

Para obtener las distribuciones observadas, se determinó la cantidad de casos (fechas de nacimiento) correspondientes a cada mes, trimestre (T) = Ene - Mar, T2 = Abr - Jun, T3 = Jul - Sep y T4 = Oct Dic) y cada semestre (S1 = Ene - Jun y S2 = Jul - Dic) a partir de los registros de LNB.

Las distribuciones esperadas por mes, trimestre y semestre se obtuvieron multiplicando la frecuencia relativa esperada (obtenida de los datos del INE) por el tamaño de los registros de LNB ( $n=183$ ).

Para calcular la significancia estadística (p) de las diferencias entre la distribuciones observadas y esperadas por mes y trimestre de nacimiento, considerando que las observaciones (fechas de nacimiento) son independientes entre sí y las categorías meses y trimestres mutuamente excluyentes, se usó el método de $\chi^{2}$ (Chi cuadrado) (Thomas et al., 2005).

En el caso de las distribuciones por semestre de nacimiento, ya que la variable "semestre de nacimiento" es dicotómica, el valor de p se calculó como la probabilidad acumulada de la frecuencia observada en el segunde semestre (S2) usando una distribución binomial, donde la probabilidad de éxitos está dada por la frecuencia relativa esperada para S2 y el número de ensayos es igual al valor de $\mathrm{n}$.

El tamaño del efecto se obtuvo de dos formas: como la diferencia relativa entre la frecuencia esperada y la observada para cada uno de los meses, trimestres y semestres estudiados y como el riesgo 
relativo entre periodos, entendiendo riesgo como la probabilidad de llegar a jugar en LNB.

\section{Resultados}

Las distribuciones por semestre (S1 y S2), esperada y observada, de las fechas de nacimiento de los jugadores participantes en LNB en la temporada 20192020 se muestran en la siguiente tabla $(n=183)$ :

Tabla 1

Distribuciones Esperadas (E) y Observada (O) por semestre de nacimiento.

\begin{tabular}{cccc}
\cline { 2 - 3 } & S1 & S2 & \\
\hline$O$ & 114 & 69 & $p=0,0002$ \\
\hline$O(\%)$ & 62,3 & 37,7 & \\
\hline$E$ & 90 & 93 & \\
\hline$E(\%)$ & 49,0 & 51,0 & \\
\hline
\end{tabular}

Fuente: Elaboración propia.

La magnitud del efecto del EER, expresado como la diferencia relativa entre los valores observadosy esperados para cada semestre, se muestran en la siguiente figura:

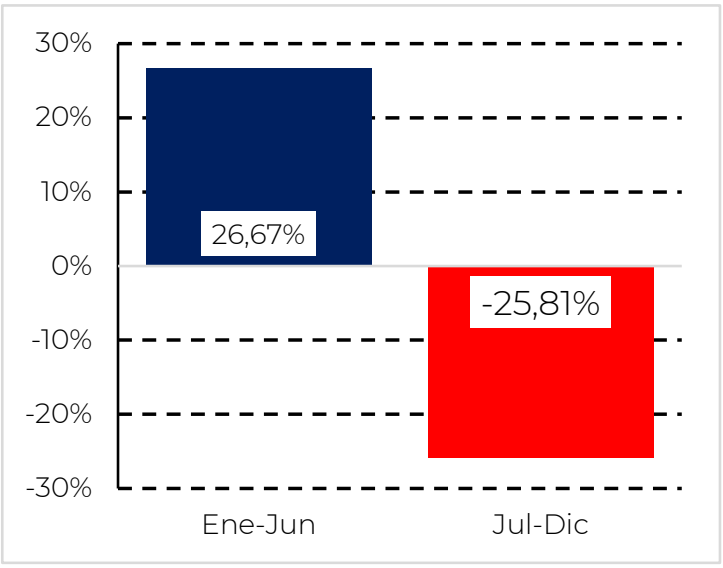

Figura 7. Magnitud del efecto del EER por semestre de nacimiento.

Fuente: Elaboración propia
Los riesgos relativos, entre los nacidos en S1 y S2 son los siguientes:

Tabla 2

Riegos Relativos por semestre de nacimiento (Fila/Columna).

\begin{tabular}{ccc}
\cline { 2 - 3 } & /S2 & /Año \\
\hline S1/ & 1,719 & 1,271 \\
\hline S2/ & 1,000 & 0,739 \\
\hline
\end{tabular}

Fuente: Elaboración propia. 
Las distribuciones por trimestre (T7, T2, T3 y T4), esperada y observada, se muestran en la siguiente tabla $(n=183)$ :

Tabla 3

Distribuciones Esperadas (E) y Observada (O) por trimestre de nacimiento.

\begin{tabular}{|c|c|c|c|c|c|}
\hline & $\mathrm{T} 7$ & T2 & T3 & T4 & \\
\hline 0 & 57 & 57 & 38 & 31 & \multirow{4}{*}{$\begin{array}{c}\chi 2=13,38 \\
P=0,0039\end{array}$} \\
\hline$O(\%)$ & 31,1 & 31,1 & 20,8 & 16,9 & \\
\hline E & 45 & 45 & 47 & 46 & \\
\hline$E(\%)$ & 24,6 & 24,4 & 25,7 & 25,3 & \\
\hline
\end{tabular}

Fuente: Elaboración propia.
La magnitud del efecto del EER, expresado como la diferencia relativa entre los valores observados y esperados para cada trimestre, se muestran en la siguiente figura:

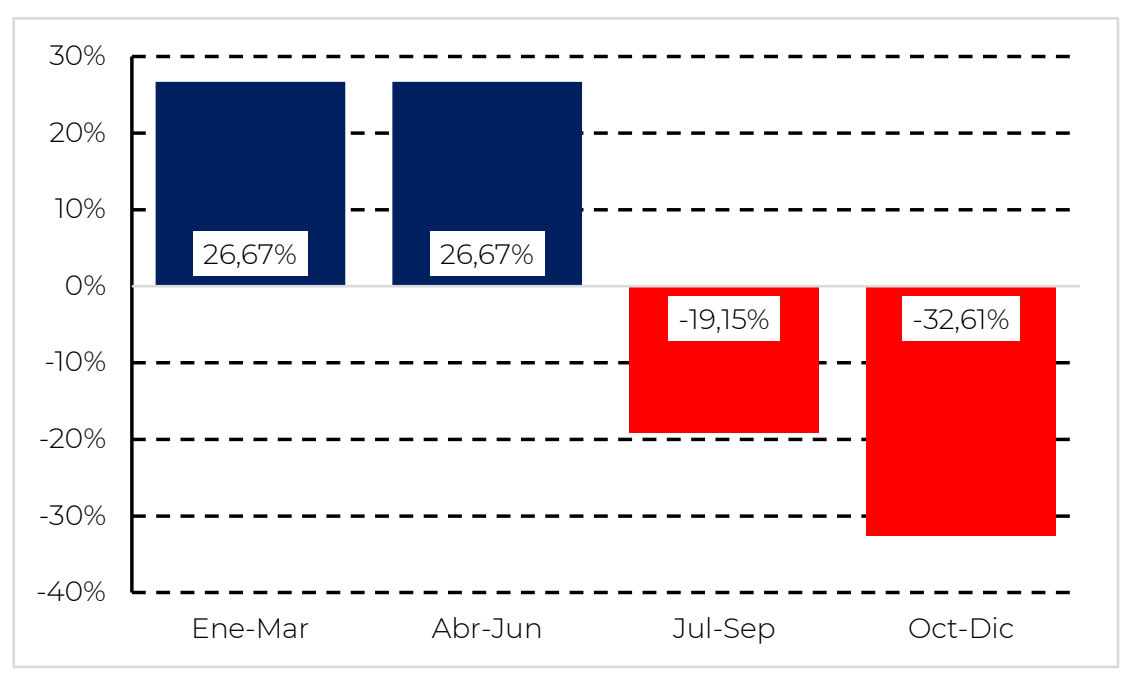

Figura 2. Magnitud del efecto del EER por trimestre de nacimiento. Fuente: Elaboración propia.

Los riesgos relativos, entre los nacidos en los diferentes trimestres son los siguientes:

Tabla 4

Riegos Relativos por trimestre de nacimiento (Fila/Columna).

\begin{tabular}{ccccc}
\cline { 2 - 5 } & /T2 & /T3 & /T4 & /Año \\
\hline T1/ & 0,992 & 1,567 & 1,890 & 1,266 \\
\hline T2/ & 1,000 & 1,580 & 1,905 & 1,276 \\
\hline T3/ & & 1,000 & 1,206 & 0,808 \\
\hline T4/ & & & 1,000 & 0,670 \\
\hline
\end{tabular}

Fuente: Elaboración propia.
Las distribuciones por mes, esperada y observada, se muestran en la siguiente tabla ( $\mathrm{n}=183$ ): 
Salinas, H. (2021). Efecto de edad relativa en la Liga Nacional de Básquetbol de Chile (LNB). Revista Ciencias de la Actividad Física UCM, N²2(1), enero-junio, 1-11. DOI: http://doi.org/10.29035/rcaf.22.1.7

Tabla 5

Distribuciones Esperadas (E) y Observada (O) por mes de nacimiento.

\begin{tabular}{|c|c|c|c|c|c|c|c|c|c|c|c|c|c|}
\hline & Ene & Feb & Mar & Abr & May & Jun & Jul & Ago & Sep & Oct & Nov & Dic & \\
\hline 0 & 25 & 15 & 17 & 19 & 21 & 17 & 17 & 11 & 10 & 10 & 14 & 7 & \multirow{4}{*}{$\begin{array}{l}\chi 2=20,04 \\
p=0,0448\end{array}$} \\
\hline ○ (\%) & 13,7 & 8,2 & 9,3 & 10,4 & 11,5 & 9,3 & 9,3 & 6,0 & 5,5 & 5,5 & 7,7 & 3,8 & \\
\hline E & 16 & 14 & 15 & 15 & 15 & 15 & 16 & 16 & 16 & 16 & 15 & 15 & \\
\hline E (\%) & 8,7 & 7,6 & 8,3 & 8,0 & 8,2 & 8,2 & 8,4 & 8,5 & 8,8 & 8,8 & 8,3 & 8,2 & \\
\hline
\end{tabular}

Fuente: Elaboración propia.

La magnitud del efecto del EER, como diferencia re-

lativa entre los valores observados y esperados para

cada mes se muestran en la siguiente figura:

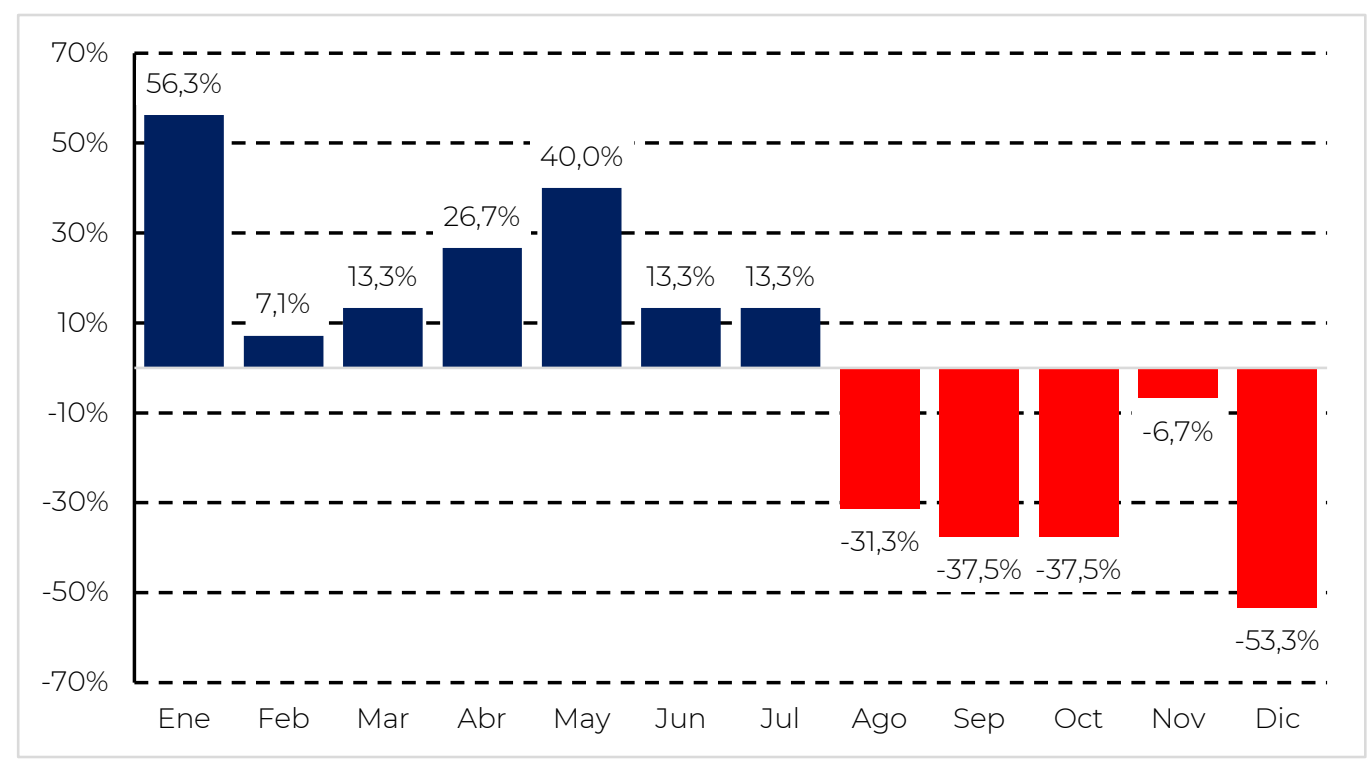

Figura 3. Magnitud del efecto del EER por mes de nacimiento.

Fuente: Elaboración propia. 
Los riesgos relativos, entre los nacidos en los diferen-

tes meses son los siguientes:

Tabla 6

Riegos Relativos por mes de nacimiento (Fila/ Columna).

\begin{tabular}{|c|c|c|c|c|c|c|c|c|c|c|c|c|}
\hline & /Feb & /Mar & /Abr & /May & /Jun & /Jul & /Ago & /Sep & /Oct & /Nov & /Dic & /Año \\
\hline Ene/ & 1,458 & 1,411 & 1,207 & 1,125 & 1,397 & 1,420 & 2,223 & 2,545 & 2,540 & 1,704 & 3,369 & 1,574 \\
\hline Feb/ & 1,000 & 0,967 & 0,827 & 0,772 & 0,958 & 0,974 & 1,524 & 1,745 & 1,742 & 1,168 & 2,310 & 1,079 \\
\hline Mar/ & & 1,000 & 0,855 & 0,798 & 0,990 & 1,006 & 1,576 & 1,804 & 1,801 & 1,208 & 2,388 & 1,116 \\
\hline Abr/ & & & 1,000 & 0,933 & 1,158 & 1,177 & 1,842 & 2,110 & 2,105 & 1,412 & 2,792 & 1,305 \\
\hline May/ & & & & 1,000 & 1,242 & 1,262 & 1,976 & 2,262 & 2,258 & 1,514 & 2,994 & 1,399 \\
\hline Jun/ & & & & & 1,000 & 1,016 & 1,591 & 1,822 & 1,818 & 1,219 & 2,471 & 1,127 \\
\hline Jul/ & & & & & & 1,000 & 1,566 & 1,793 & 1,789 & 1,200 & 2,373 & 1,109 \\
\hline Ago/ & & & & & & & 1,000 & 1,145 & 1,143 & 0,767 & 1,516 & 0,708 \\
\hline Sept/ & & & & & & & & 1,000 & 0,998 & 0,669 & 1,324 & 0,618 \\
\hline Oct/ & & & & & & & & & 1,000 & 0,671 & 1,326 & 0,620 \\
\hline Nov/ & & & & & & & & & & 1,000 & 1,977 & 0,924 \\
\hline Dic/ & & & & & & & & & & & 1,000 & 0,467 \\
\hline
\end{tabular}

Fuente: Elaboración propia.

\section{Conclusión}

Aceptando que los resultados encontrados, no son producto del azar ( $p=0,020 \%, 0,389 \%$ y 4,477\% para las distribuciones, semestrales, trimestrales y mensuales respectivamente), se debe concluir que el sistema de formación de jugadores de baloncesto del cual provienen los jugadores chilenos actualmente en la LNB, privilegia a los nacidos durante el primer semestre del año, especialmente a los nacidos en enero, por sobre los nacidos en el segundo semestre, sobre todo si estos últimos nacieron durante el cuarto trimestre o en diciembre.

Un análisis de riesgo relativo, entendiendo "riesgo" como la probabilidad de llegar a jugar en LNB, arroja que los nacidos en S1 tienen 1,72 más probabilidad que los nacidos en S2. Este valor crece a 1,89 al comparar a los nacidos en T7y T4y al hacer un análisis mensual, indica que los nacidos en el mes de enero tienen 3,37 veces más probabilidades de llegar a LNB que los nacidos en el mes de diciembre.

\section{Discusión}

Estos resultados en ningún caso indican algún tipo de intencionalidad por parte de entrenadores o dirigentes, sino que manifiestan la natural ventaja que, en categorías formativas, un jugador de mayor edad tiene en un deporte como el baloncesto. Numerosos estudios han mostrado que los deportistas nacidos en los últimos meses del año tienden, durante la adolescencia, a tener menor maduración física que los nacidos durante la primera parte del año (Isorna et al., 2014).

Por ejemplo, un niño de 10 años, dentro del 5\% más pequeño, pesará 22 kilos y medirá $126 \mathrm{~cm}$ aproximadamente, mientras que un niño de 11 años dentro del 95\% más alto para su edad, pesará 49 kg y medirá $154 \mathrm{~cm}$ aproximadamente. Así, un niño pequeño de 10 años podría ser $28 \mathrm{~cm}$. más bajo y $27 \mathrm{~kg}$ más liviano que un madurador temprano de 11 años, con una diferencia de edad de sólo un año (Helsen et al., 2005). 
Al ser el baloncesto un deporte donde cualidades físicas como la estatura, fuerza y velocidad, dependientes de la edad del niño, son determinantes, es muy probable que un jugador de mayor edad tenga ventaja sobre sus compañeros menores, aún dentro de la misma categoría, por el simple hecho de ser mayor (Mujika et al., 2009, Leite et al., 2013). Esta situación podría verse acentuada por la división en grupos que abarcan periodos de dos años, como es el caso chileno, donde, en una categoría sub-12, un niño de 12 años podría ser un 20\% mayor que un niño de 10 años.

Esta ventaja física determinará un mayor rendimiento deportivo que, en un contexto competitivo, implicará mayor tiempo de juego y promoción a niveles superiores de competencia, con el consecuente reconocimiento del entorno y exposición a más y mejor entrenamiento, factores que, en conjunto, determinan un incremento en la diferencia de rendimiento que pueden tener los niños nacidos en los primeros meses del año respecto de los nacidos más tarde, quienes presentan mayores probabilidades de abandono, sobre todo si son maduradores tardíos (Leite et al., 2013).

Este efecto sobre ambos grupos de jugadores determina que una mayor proporción de jugadores nacidos al inicio del año llegue a estar disponible para ser seleccionados en los equipos adultos de elite, lo que explicaría este fenómeno en dicho nivel donde la ventaja física, debido a la mayor edad, no es un factor.

Además de las fechas de corte, la literatura propone otras posibles explicaciones al EER, entre las que destaca el efecto del clima como fenómeno estacional de ciclo anual. Estas circunstancias, podrían ser relevantes para el desarrollo de habilidades deportivas en niños. Por ejemplo, si un clima tibio coincide con momentos relevantes para la adquisición de habilidades motrices y actividades al aire libre, promueven su desarrollo; los niños nacidos en ciertas épocas del año presentarían ventajas sobre los nacidos en otros momentos. Esto crearía una falsa idea de un EER asociado a la fecha de corte de los grupos de competencia. Sin embargo, los meses de diciembre y enero, están en los extremos opuestos del año de competición en baloncesto y presentan claramente el EER, y al mismo tiempo, son muy similares climáticamente, lo que indicaría que la estacionalidad climática no tiene un efecto importante (Musch \& Grondin, 2001).

EI EER representa un potencial desperdicio de talento debido al abandono temprano de maduradores tardíos y jugadores nacidos hacia fin de año. Existen estudios que señalan que deportistas con un desarrollo físico tardío, desarrollan de mejor forma aspectos técnicos y tácticos durante su etapa de formación, como una forma de contrarrestar su menor potencial físico, lo que podría generar un mayor rendimiento en niveles superiores (Isorna et al., 2014).

Para evitar este fenómeno, es indispensable que los responsables de la promoción del talento deportivo (entrenadores, padres y directivos), conozcan el fenómeno y consideren la edad cronológica y alguna estimación del nivel de maduración de sus jugadores y sus efectos sobre el rendimiento, de modo de considerar este factor a la hora de promover o descartar jugadores de los niveles superiores de competición. En este sentido, se sugiere la propuesta de Sherar et al. (2005) que permite una estimación de la edad biológica mediante el uso de variables antropométricas simples.

Un estudio realizado en Holanda indica que el sesgo en la selección de talentos en fútbol se disminuye o elimina si la información respecto de la edad de los jugadores es presentada adecuadamente. En este estudio, 25 buscadores de talentos del club PSV Eindhoven, observaron videos de partidos de fútbol reducido de jugadores Sub-11 y ordenaron a los jugadores basados en su potencial deportivo. Los buscadores de talentos fueron asignados a uno de tres grupos, cada uno con diferente información respecto de las edades de los jugadores: un primer grupo sin información, el segundo conocía la fecha de nacimiento de los jugadores y el tercero conocía que los números en las camisetas correspondían a la edad relativa de los jugadores. Los resultados revelaron un importante sesgo en el primer y segundo grupo, el cual fue eliminado cuando los 
observadores conocían que los números en las camisetas correspondían a la edad relativa de los jugadores (Mann \& Pleun, 2017).

Otra posible medida, esta vez a nivel de organización de competencias de divisiones formativas, consiste en variar las fechas de corte al largo del año competitivo. Estudios indican que el EER se traslada junto con la fecha de corte (Mujika et al., 2009), por lo que sucesivas modificaciones de esta fecha a lo largo del año competitivo, podrían "repartir" entre más jugadores la ventaja de cumplir años justo después de ella. Por ejemplo, en vez de realizar torneos anuales manteniendo estática la fecha de corte (1 de enero) durante el año competitivo, podrían organizarse 3 torneos con fechas de corte avanzando 4 meses en cada uno, (1 de enero, 1 de mayo y 1 de septiembre) dando a todos la oportunidad de ser los mayores del grupo etario.

El trato injusto hacia algunos jugadores generado por el EER tiene consecuencias a nivel personal como desmotivación y cese de la práctica deportiva

\section{REFERENCIAS BIBLIOGRÁFICAS}

Addona, V., \& Yates, P. (2010). A Closer Look at the Relative Age Effect in the National Hockey League. Journal of Quantitative Analysis in Sports, 6(4). https://doi.org/10.2202/15590410.1227

Albuquerque, M., Franchini, E., Lage, G., Da Costa, V., Costa, I., \& Malloy-Diniz, L. (2015). The relative age effect in combat sports: An analysis of olympico Judo Athletes, 1964-2012. Perceptual \& Motor Skills: Physical Development \& Measurement, 127(1), 300308. https://doi.org/10.2466/10.PMS.121c15×2

Barnsley, R., \& Thompson, A. (1988). Birthdate and success in minor hockey: The key to the NHL. Revue canadienne des sciences du comportement, 20(2), 167-176. https://doi.org/10.1037/h0079927 e institucional debido a la potencial pérdida de talento deportivo. Llamar la atención de entrenadores y directivos respecto de la existencia y consecuencias del fenómeno es el primer paso para remediarlo. Si además se implementan masiva y sistemáticamente medidas que permitan conocer el nivel de maduración biológica de los jugadores y se toman decisiones de acuerdo con ella, se habrá dado un gran paso hacia mejorar el trabajo de formación de jugadores. Finalmente, un cambio hacia una estructura de competencia más acorde a las necesidades de los jugadores en formación podría, en el largo plazo, mejorar el nivel global del baloncesto chileno o, al menos, tener niños y jóvenes más felices de practicar baloncesto.

Delorme, N., Boiché, J., \& Raspaud, M. (2009). The Relative Age Effect: The French Case. Research Quarterly for Exercise \& Sport, $80(2)$,

336-344. https://doi.org/10.1080/02701367.2009.10599 568

Delorme, N., Boiché, J., \& Raspaud, M. (2010). Relative Age Effect in Elite Sports: Methodological Bias or Real Discrimination? European Journal of Sport Science, 10(2), 91-96. https://doi.org/10.1080/17461390903271584

Delorme, N., Chalabaev, A., \& Raspaud, M. (2011). Relative Age is Associated with Sport Dropout: Evidence from Youth Categories of French Basketball. Scandinavian Journal of Medicine and Science in Sports, 27(1), 120128. https://doi.org/10.1111/j.16000838.2009.01060.x 
Febachile. (2019). Equipos LNB DIRECTV 2019-2020. http://clnb.web.geniussports.com/competitions/?WHurl=\%2Fcompetition\%2F25729\%2Fteams\%3F

Gutierrez, D., Pastor, J., Gonzalez, S., \& Contreras, O. (2010). The relative age effect in youth soccer players from Spain. Journal of Sports Science and Medicine, 9(2), 190-198. https://www.ncbi.nlm.nih.gov/pmc/articles/PMC3761747/

Helsen, W., Van Winckel, J., \& Williams, M. (2005). The relative age effect in youth soccer across Europe. Journal of Sports Science, 23(6), 629-636.

https://doi.org/10.1080/02640410400021310

Ibáñez, S., Mazo, A., Nascimento, J., \& García-Rubio, J. (2018). The Relative Age Effect in under-18 basketball: Effects on performance according to playing position. Plos One, 13(7), e0200408. https://doi.org/10.1371/journal.pone.0200408

INE (s.f.). Estadísticas Vitales. https://www.ine.cl/estadisticas/sociales/demografia-y-vitales

Isorna, M., Vaquero, R., Vinuesa, J., \& Rial, A. (2014). El efecto de la edad relativa en la participación en el Plan nacional de tecnificación deportiva y la consecución de grandes éxitos deportivos en piragüismo. Apunts. Educación Física y Deportes, (175), 46-53. https://doi.org/10.5672/apunts.20140983.es.(2014/1).115.04

Leite, N., Borges, J., Santos, S., \& Sampaio, J. (2013). The relative age effect in school and federative sport in basketball. Revista de Psicología del Deporte, 22(1), 219-223. https://www.redalyc.org/pdf/2351/235127552025.pdf

Loffing, F., Schorer, J., \& Cobley, S. (2010). Relative Age Effects are a developmental problem in tennis: but not necessarily when you're lefthanded! High Ability Studies, 27(1), 19-25. https://doi.org/10.1080/13598139.2010.48808 4
Lorenzo, A. (2002). La detección del talento en los deportes colectivos. Kronos, 7(1), 15-23. https://abacus.universidadeuropea.es/bitstream/handle/11268/1421/kronos_1_3.pdf?sequence=1

Mann, D., \& Pleun J. M. A. (2017). Age-ordered shirt numbering reduces the selection bias associated with the relative age effect. Journal of Sports Sciences, 35(8), 784-790. https://doi.org/10.1080/02640414.2016.11895 88

Manonelles, P., Álvarez, J., Coloma, M., Sainz de Aja, C., Corona, P., \& Gimenez, L. (2003). Edad Cronológica como factor de elección de jugadores de las selecciones españolas de baloncesto de formación. Archivos de Medicina del Deporte, 20(96), 321-328. https://pesquisa.bvsalud.org/portal/resource/pt/ibc-29238

Mujika, I., Vaeyens, R., Matthys, S., Santiesteban, J., Goiriena, J., \& Philippaerts, R. (2009). The relative age effect in a professional football club setting. Journal of Sports Sciences, 27(11), 1153-1158. https://doi.org/10.1080/02640410903220328

Musch, J., \& Grondin, S. (2001). Unequal competition as an impediment to personal development: A review of the relative age effect in sport. Developmental Review, 27(2), 147-167. https://doi.org/10.1006/drev.2000.0516

Saavedra, M., Gutiérrez, O., Galatti, L., \& Fernández, J. (2015). Efecto de la edad relativa en los mundiales de baloncesto FIBA en categorias inferiores (1979-2011). Cuadernos de Psicología del Deporte, 15(3), 237-242. https://www.redalyc.org/pdf/2270/227042879028.pdf 
Schorer, J., Wattie, N., \& Baker, J. (2013). A New Dimension to Relative Age Effects: Constant Year Effects in German Youth Handball. Plos One, 8(5), e60336. https://doi.org/10.1371/annotation/346754d7-87e5-4e5f-90c8$86 a 23632227 a$

Sherar, L., Mirwald, R., Baxter-Jones, A., \& Thomis, M. (2005). Prediction Of Adult Height Using Maturity-Based Cumulative Height Velocity Curves. The Journal of Pediatrics, 147(4), 508-514.

https://doi.org/10.1016/j.jpeds.2005.04.041

Thomas, J., Nelson, J., \& Silverman, S. (2005). Research Methods in Physical Activity. Human Kinetics.
Úbeda-Pastor, V., Guerrero-Jiménez, P., \& LlanaBelloch, S. (2020). Efecto de la Edad Relativa en Cinco Ligas Europeas de Fútbol Profesional. Kronos, 19(1), 1-7. https://revistakronos.info/articulo/efecto-de-la-edadrelativa-en-cinco-ligas-europeas-de-futbolprofesional-2773-sa-H5ef26635b90c2

Werneck, F., Coelho, E., de Oliveira, H., Ribeiro Júnior, D., Almas, S., de Lima, J., ... Figueiredo, A. (2016). Relative age effect in Olympic basketball athletes. Science \& Sports, 37(3), 158-161.

https://doi.org/10.1016/j.scispo.2015.08.004

\section{Dirección para correspondencia}

Hernán Andrés Salinas Molina

Ingeniero Civil (PUC), Licenciado en Ciencias de la Actividad Física (USACH), Máster en Rendimiento Deportivo (INEFC).

Docente Facultad de Educación y Ciencias Sociales

Universidad Nacional Andrés Bello, Santiago, Chile

Institute for Globally Distributed Open Research and Education

(IGDORE)

ORCID ID: https://orcid.org/0000-0002-8846-8104

Dirección Postal: Bremen 1120, Ñuñoa

Santiago, Chile.

Contacto:

hasalina@uc.cl

Recibido: 28-10-2020

Aceptado: 28-04-2021 